\title{
Idiopathic Pulmonary Hemosiderosis in an Adult with Unusual Radiologic Features
}

\author{
${ }^{1}$ Sangeeth Kumar Kasilingam, ${ }^{2}$ Shylaja Prashanth, ${ }^{3}$ Manjunath B Govindagoudar, ${ }^{4}$ Bheemaraya Devaramani \\ ${ }^{5}$ Chaitra Channarayapatna Swamygowda
}

\begin{abstract}
Idiopathic pulmonary hemosiderosis is an exceptionally rare cause of diffuse alveolar hemorrhage that occurs primarily in infants and children. Few cases are reported in adults. Patients usually present with recurrent episodes of hemoptysis, fatigue breathlessness and severe anemia with asymptomatic periods in between. Our patient, who was diagnosed with idiopathic pulmonary hemosiderosis demonstrated diffuse ground glass opacity, focal centrilobular emphysema and multiple sub pleural cysts and few parenchymal cysts during acute episode of hemoptysis. Unusual findings in our case were sub pleural cysts, parenchymal cysts and focal centrilobular emphysema which was not documented in any case reports of idiopathic pulmonary hemosiderosis cases to that of our knowledge.
\end{abstract}

Keywords: Computed tomography high resolution, Hemorrhage, Cysts, Adults, Thorax.

How to cite this article: Kasilingam SK, Prashanth $S$, Govindagoudar MB, Devaramani B, Swamygowda CC. Idiopathic Pulmonary Hemosiderosis in an Adult with Unusual Radiologic Features. J Postgrad Med Edu Res 2014;48(4):199-200.

Source of support: Nil

Conflict of interest: None

\section{INTRODUCTION}

Diffuse alveolar hemorrhage (DAH) is a potentially catastrophic complication of myriad immune and non immune disorders. Clinical features are broad, but hemorrhage, hemoptysis, infiltrates on chest radiographs, hypoxemia and progressive respiratory insufficiency are common to diverse etiologies. A diagnosis of idiopathic pulmonary hemosiderosis can be made only when other specific causes of DAH have been reliably excluded. ${ }^{1}$

\section{PRESENTATION}

A 36-year-old woman presented to us with history of fever, cough, hemoptysis and easy fatiguability since 4 to 5 days. Fever was of moderate degree, continuous, not associated with chills and rigors. Hemoptysis was

\footnotetext{
${ }^{1,2}$ Assistant Professor, ${ }^{3}$ Senior Resident, ${ }^{4,5}$ Medical Officer ${ }^{1,3-5}$ Department of Pulmonary Medicine, ESIC MC and PGIMSR, Rajajinagar, Karnataka, India

${ }^{2}$ Department of Radiodiagnosis, ESIC MC and PGIMSR Rajajinagar, Karnataka, India
}

Corresponding Author: Sangeeth Kumar Kasilingam Assistant Professor, Department of Pulmonary Medicine ESIC MC and PGIMSR, Rajajinagar, Karnataka, India, Phone: 08023528613, e-mail: sangeethbmcmed@gmail.com massive and many episodes in a day. Following hemoptysis, she developed generalized weakness and breathlessness at rest. She has history of similar episodes since 2 years, roughly one episode every 6 months. During every episode, the patient was hospitalized due to hemoptysis and severe anemia. She has history of blood transfusion during every episode.

\section{CLINICAL EXAMINATION}

The patient was breathless at rest. She had cold peripheries. Pulse-120/minutes, blood pressure- $80 / 70 \mathrm{~mm} \mathrm{Hg}$, respiratory rate-38/minutes. $\mathrm{SpO}_{2}$ at room air was $78 \%$. The patient was admitted in intensive care unit and treated with $\mathrm{O}_{2}$ inhalation and supportive medications.

\section{LABORATORY AND RADIOGRAPHIC FINDINGS}

$\mathrm{Hb}-3.5 \mathrm{gm} / \mathrm{dl}$, total white blood cell (WBC) count $9 \times 10^{9} / \mathrm{l}$, differential count-P 82, L 12, E 2, M 4, platelet count-350 $\times 10^{9} / \mathrm{l}$, prothrombin time International normalised ratio (INR) - 1.01, activated partial thromboplastin time-25.5 seconds, RBS-86 mg/dl, blood urea $-60 \mathrm{mg} / \mathrm{dl}$, S creatinine $-0.6 \mathrm{mg} / \mathrm{dl}$, iron study: total iron $-25 \mu \mathrm{g} / \mathrm{dl}$, total iron binding capacity (TIBC) $-391 \mu \mathrm{g} / \mathrm{dl}$, transferrin—372 mg/dl, transferrin saturation- $4.7 \%$, autoantibody profilenegative, antineutraphil cystoplasmic antibody (ANCA) negative, anti glomerular basement membrane antibodynegative, tissue transglutaminase $\mathrm{IgA}-1.9 \mathrm{U} / \mathrm{ml}$ (negative). Bone marrow examination showed micronormoblastic hyperplasia. Chest $\mathrm{X}$-ray showed diffuse alveolar infiltrates. Computed tomography (CT) high resolution chest showed ground glass opacity (Fig. 1, star), honey combing (Fig. 1, solid white arrow), centrilobular emphysema involving anterior and inferior segment of right upper lobe and most of the right middle lobe (Fig. 2, white solid arrow head) and multiple sub pleural cysts (Fig. 1, solid black arrow) and few parenchymal cysts, traction bronchiectasis (Fig. 1, hollow white arrow) during acute episode of hemoptysis. CT pulmonary angiogram did not show any vascular abnormalities. Bronchoscopy did not show any endobronchial lesion. Transbronchial lung biopsy showed numerous clumps of hemosiderin laden macrophages. Pulmonary function test showed moderate restrictive defect.

\section{DIAGNOSIS AND MANAGEMENT}

A diagnosis of idiopathic pulmonary hemosiderosis was made, since all other causes for pulmonary 


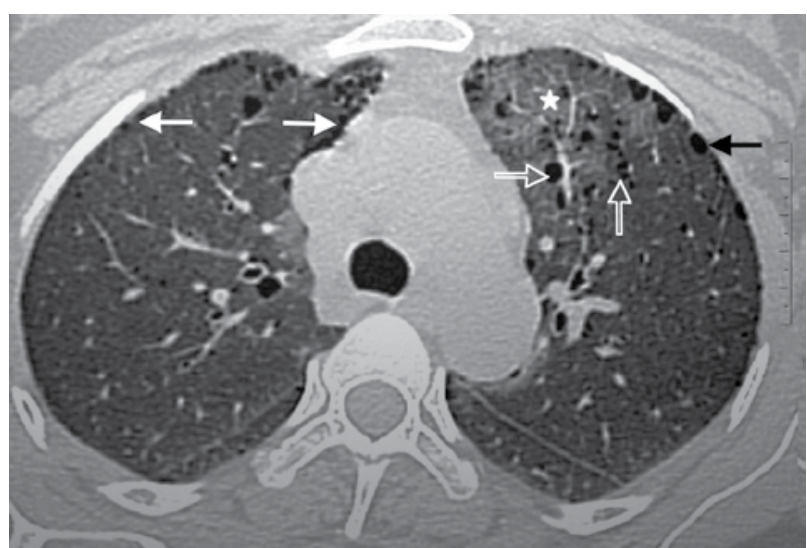

Fig. 1: Solid blackarrow—subpleural cyst, solid whitearrow-honeycombing, hollow white arrow-bronchiectasis, star-ground glass opacity

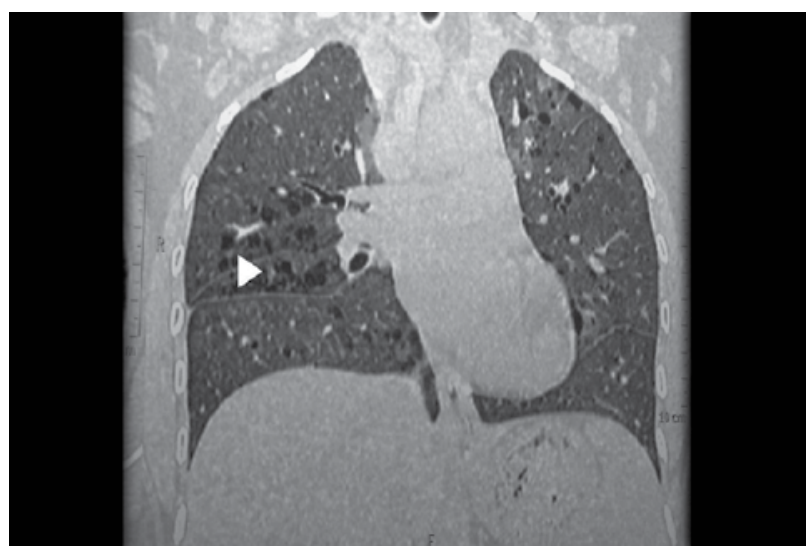

Fig. 2: Solid white arrow head—centrilobular emphysema

hemosiderosis were ruled out. The patient was treated with blood transfusion and other supportive medications. Oral prednisolone at a dose of $1 \mathrm{mg} / \mathrm{kg}$ body weight was added and later tapered and continued on maintenance dose. Follow-up high-resolution computed tomography (HRCT) lung done after 3 months showed significant resolution of ground glass opacity. However, rest of the lesions, viz. honeycombing, sub pleural cysts, traction bronchiectasis, interlobular septal thickening and centilobular emphysema persisted.

\section{DISCUSSION}

The clinical course of idiopathc pulmonary hemosiderosis is variable, but recurrent episodes of DAH over several years are characteristic. CT thorax done during these episodes usually reveals ground glass opacification representing foci of alveolar hemorrhage. ${ }^{1}$ Thickening of interlobular septae and honey combing is seen in a subset of patients who progress to pulmonary fibrosis. ${ }^{1}$ Sequelae of recurrent episodes of DAH include: pulmonary fibrosis, progressive respiratory failure and cor pulmonale. During acute episodes, chest radiographs demonstrate bilateral alveolar infiltrates. Following cessation of bleeding, chest radiographs may normalize within 1 to 2 weeks. Reticulonodular infiltrates may be observed as the process is resolving or with recurrent episodes. ${ }^{1}$ With repeated bleeding episodes, seen typically in primary pulmonary hemosiderosis, the interstitial shadowing fails to clear completely, and the patient may be left with a permanent, fine reticulonodular ${ }^{2}$ or nodular $(2-3 \mathrm{~mm})^{3}$ opacity that tends to increase in profusion with each acute episode.

HRCT descriptions of idiopathic pulmonary hemosiderosis (IPH) in adults are uncommon. Cheah et $\mathrm{al}^{4}$ described the appearances of recurrent pulmonary hemorrhage in six adults, including diffuse ground glass opacities and consolidation, increased interstitial lines consistent with fibrosis, and centrilobular nodules in two patients. Buschman and Ballard described the HRCT changes in a 53-year-old woman with IPH. Severe fibrotic changes with progressive massive fibrosis were noted in the upper lobes, with honeycombing in the posterior segments of both lowerlobes. ${ }^{5}$ Honey comb cysts in two cases of idiopathic pulmonary hemosiderosis have also been described. ${ }^{6}$ Unusual findings in our case was centrilobular emphysema, sub pleural and few parenchymal cysts in our case which was not documented in any case reports of idiopathic pulmonary hemosiderosis cases to that of our knowledge. However, the cause for centrilobular emphysema could not be ascertained as the patient did not have any history of smoking. The pathogenesis of appearance of lung cysts and subpleural cyst although not clear, could possibly be due to lung destruction secondary to hemosiderin deposition.

\section{CONCLUSION}

Centrilobular emphysema, parenchymal and sub pleural cysts could probably represent other radiological presentations of idiopathic pulmonary hemosiderosis apart from ground glass opacification, honeycombing, centilobular nodules and interstitial septal thickening.

\section{REFERENCES}

1. Lynch JP III, Leatherman JW. Alveolar hemorrhage syndromes. In: Fishman AP, Elias JA, Fishman JA, editors. Fishman's Pulmonary Diseases and Disorders. 4th ed. United States 07. p. 1281-1297.

2. Soergel KH, Sommers SC. Idiopathic pulmonary hemosiderosis and related syndromes. Am J Med 1962;32(4):499-511.

3. Cheah FK, Sheppard MN, Hansell DM. Computed tomography of diffuse pulmonary haemorrhage with pathological correlation. Clin Radiol 1993;48(2):89-93.

4. Cheah FK, Sheppard MN, Hansell DM. Computed tomography of diffuse pulmonary haemorrhage with pathological correlation. Clin Radiol 1993;48(2):89-93.

5. Buschman DL, Ballard R. Progressive massive fibrosis associated with idiopathic pulmonary hemosiderosis. Chest 1993;104(1):293-295.

6. Harte S, Mc Nicholas WT, Donnelly SC, Dodd JD. Honey comb cysts in idiopathic pulmonary hemosiderosis: high resolution CT appearances in two adults. Br J Radiol 2008 Dec;81(972):e295-e298. 